\title{
Hybrid Therapy as a Last Resort in the Treatment of Complex Ventricular Tachyarrhythmias
}

\author{
Pekka Raatikainen \\ Division of Cardiology, Heart and Lung Center, Helsinki University Hospital, Helsinki, Finland
}

The cornerstones in the treatment of life-threatening ventricular tachyarrhythmias (VA) and prevention of sudden arrhythmic death are appropriate management of the underlying diseases and implantable cardioverter defibrillator therapy. In addition, the treatment of VA commonly requests the use of antiarrhythmic drug therapy, revascularization, percutaneous catheter ablation, and/or arrhythmia surgery [1-3]. Over the last decades, remarkable progress has been made in the field of VA therapy. Several trials have demonstrated that cardioverter defibrillator therapy provides safe and efficacious means for preventing arrhythmic death among subjects with elevated risk of VA [3]. At the same time, catheter ablation has undergone rapid progress with major advantages in the technology used to treat VA. The introduction of electroanatomic mapping systems, open-irrigated ablation catheters, and other technological advancements have markedly improved the efficacy and safety of VA ablation. Despite these innovations, the treatment of VA is still challenging, and arrhythmia recurrences are common after catheter ablation.

In this issue, Haanschoten et al. [4] report the results of a small study evaluating the feasibility of surgical openchest endo- and epicardial VA ablation in patients with previously failed percutaneous catheter ablation. Hybrid ablation was performed under extracorporeal circulation

\section{KARGER}

(๑) 2020 S. Karger AG, Basel

E-Mail karger@karger.com

www.karger.com/crd
(ECC) support in 5 male patients (age 62-76 years). Three of them had prior myocardial infarction, and 2 had nonischemic cardiomyopathy. Arrhythmia surgery is an old concept. As early as in 1959, Couch [5] reported that resection of postinfarction left ventricular aneurysm eliminated recurrent ventricular tachycardia (VT). In the 1970s, Mark Josephson's group developed the technique of map-guided surgical subendocardial resection $[6,7]$. These findings inspired to the development of percutaneous catheter ablation techniques for the treatment of drug-resistant VA. It has been shown that late potentials, low-voltage areas, fragmented signals, and local abnormal ventricular activity recorded during normal rhythm or pacing are valuable surrogates of VA circuits, and ablation of these areas can eliminate VA $[1,2,8]$. After the publication of these fundamental studies, the use of percutaneous catheter ablation for VA has increased rapidly and has replaced arrhythmia surgery as the initial therapy for drug-resistant VA. According to the contemporary ventricular tachyarrhythmia management guidelines, catheter ablation should be considered for treatment of VA when drugs are ineffective or cause unacceptable adverse effects [1-3].

It is important to note that percutaneous catheter ablation of complex VA has several limitations. First, detailed mapping of the VT circuit is not possible in hemodynam- 
ically unstable patients without circulatory support. In such cases, substrate-based ablation during normal rhythm or pacing has proven useful in reducing VA recurrences [2]. Unfortunately, particularly in patients with nonischemic cardiomyopathy, conventional endocardial catheter ablation has limited efficacy as the predominant arrhythmia circuits are commonly epicardial. According to a recent meta-analysis, in such cases, combined endoand epicardial ablation is associated with a lower risk of VA recurrence and mortality than endocardial ablation alone [9]. Epicardial procedures are usually performed in the cardiac electrophysiology laboratory. Access into the pericardial space is attained via subxiphoid puncture as originally described by Sosa et al. [10]. However, in patients with prior open-heart surgery, percutaneous pericardial access is usually not achievable, and occasionally the presence of inflammatory pericardial adhesions makes pericardial puncture impossible. Other concerns related to percutaneous epicardial ablation include localization of the coronary arteries, phrenic nerve, or epicardial fat in the target area [11]. In the current study, several steps were used to overcome these limitations. All procedures were performed in a hybrid electrophysiology laboratory equipped with a state-of-the-art electroanatomic 3D mapping system, and high-density mapping with a multi-electrode catheter was used to guide the ablations. The open-chest setting provided direct access to the pericardial space and allowed mobilization of the heart as well as displacement or removal of epicardial fat, the phrenic nerve, or coronary artery from the target area. The ECC support allowed detailed mapping of the arrhythmia circuit also during hemodynamically instable VA.

What can we recommend at the present time? According to the results of the current study, hybrid therapy is promising as acute success was achieved in all patients. However, the results also indicate that the risk of severe procedure-related complications is high. In 1 patient, median sternotomy caused severe damage to a venous graft, and the patient died. Hence, it is apparent that hybrid therapy should not be used as first-line therapy for drugresistant VA. On the other hand, there is no doubt that in selected patients with previously failed percutaneous endo- and epicardial catheter ablation, hybrid therapy can be lifesaving. In patients with prior cardiac surgery, percutaneous pericardial access is challenging, and clinicians often opt for surgical access. Our ability to detect disrupted electrical signals related to the VA circuit is highly dependent on the quality of the local electrograms. In the light of prior [12] and current data, it is likely that high-density electroanatomic mapping with a multipolar catheter helps recognize and eliminate regions that are critical to the maintenance of the VA. Finally, although the definite benefit of ECC support remains to be established, these findings suggest that hemodynamic support plays an important role in complex VA ablation.

Several important issues need further evaluation. First, compared to conventional VA ablation, the risk of severe complications related to the ECC supported hybrid ablation is high. Hence, when planning hybrid ablation, the other therapeutic options must be carefully evaluated, and the expected success rate of the hybrid procedure needs to be weighed against the risk of serious complications. Second, the impact of preoperative imaging on the selection of patients who are likely to benefit from hybrid therapy must be carefully evaluated. Preoperative imaging of the arrhythmia substrate by computed tomography or cardiac magnetic resonance imaging may provide essential information on the location of the scar and differentiate between endo- and epicardial arrhythmia substrate. Third, it remains to be established whether the hybrid approach improves the long-term success of VA ablation. According to the contemporary guidelines, the decision between conventional versus hybrid endo- and epicardial mapping and ablation is left to the evaluation of the treating physicians. In this process, the experience of the team plays a central role. Finally, it is likely that the need for hybrid operations will increase. This should be taken into account when building new electrophysiology laboratories and training electrophysiologists and cardiac surgeons.

In summary, the findings of Haanschoten et al. [4] indicate that hybrid therapy has an important role in the treatment of complex VA. However, due to high risk of serious complications, it should be considered a last resort and only be performed in highly experienced centers with close collaboration between electrophysiologists and cardiac surgeons.

\section{Acknowledgments}

P.R. is supported by the Foundation for Cardiovascular Research, Helsinki, Finland.

\section{Disclosure Statement}

P.R. has acted as a consultant for Biosense Webster and Stereotaxis in the management of complex atrial and ventricular arrhythmias.
96

Cardiology 2020;145:95-97

DOI: $10.1159 / 000505178$
Raatikainen 


\section{References}

1 Al-Khatib SM, Stevenson WG, Ackerman MJ, Bryant WJ, Callans DJ, Curtis AB, et al. 2017 AHA/ACC/HRS guideline for management of patients with ventricular arrhythmias and the prevention of sudden cardiac death: A Report of the American College of Cardiology/ American Heart Association Task Force on Clinical Practice Guidelines and the Heart Rhythm Society. Heart Rhythm. 2018 Oct; 15(10):e73-189.

2 Pedersen CT, Kay GN, Kalman J, Borggrefe M, Della-Bella P, Dickfeld T, et al.; EP-Europace, UK. EHRA/HRS/APHRS expert consensus on ventricular arrhythmias. Heart Rhythm. 2014 Oct;11(10):e166-96.

3 Priori SG, Blomström-Lundqvist C, Mazzanti A, Blom N, Borggrefe M, Camm J, et al.; Task Force for the Management of Patients with Ventricular Arrhythmias and the Prevention of Sudden Cardiac Death of the European Society of Cardiology (ESC). 2015 ESC Guidelines for the management of patients with ventricular arrhythmias and the prevention of sudden cardiac death: The Task Force for the
Management of Patients with Ventricular Arrhythmias and the Prevention of Sudden Cardiac Death of the European Society of Cardiology (ESC)Endorsed by: Association for European Paediatric and Congenital Cardiology (AEPC). Europace. 2015 Nov;17(11):160187.

4 Haanschoten DM, Adiyaman A, Smit JJJ, Delnoy PPHM, Ramdat Misier AR, Porta F, et al. Hybrid Ventricular Tachycardia Ablation after Failed Percutaneous Endocardial and Epicardial Ablation. Cardiology. DOI: 10.1159/ 000503251.

5 Couch OA Jr. Cardiac aneurysm with ventricular tachycardia and subsequent excision of aneurysm; case report. Circulation. 1959 Aug; 20(2):251-3.

6 Horowitz LN, Harken AH, Kastor JA, Josephson ME. Ventricular resection guided by epicardial and endocardial mapping for treatment of recurrent ventricular tachycardia. $\mathrm{N}$ Engl J Med. 1980 Mar;302(11):589-93.

7 Josephson ME, Harken AH, Horowitz LN. Endocardial excision: a new surgical technique for the treatment of recurrent ventricular tachycardia. Circulation. 1979 Dec;60(7): 1430-9.
8 Santangeli P, Marchlinski FE. Substrate mapping for unstable ventricular tachycardia. Heart Rhythm. 2016 Feb;13(2):569-83.

9 Romero J, Cerrud-Rodriguez RC, Di Biase L, Diaz JC, Alviz I, Grupposo V, et al. Combined Endocardial-Epicardial Versus Endocardial Catheter Ablation Alone for Ventricular Tachycardia in Structural Heart Disease: A Systematic Review and Meta-Analysis. JACC Clin Electrophysiol. 2019 Jan;5(1):13-24.

10 Sosa E, Scanavacca M, d'Avila A, Pilleggi F. A new technique to perform epicardial mapping in the electrophysiology laboratory. J Cardiovasc Electrophysiol. 1996 Jun;7(6):531-6.

11 Maccabelli G, Mizuno H, Della Bella P. Epicardial ablation for ventricular tachycardia. Indian Pacing Electrophysiol J. 2012 Nov; 12(6):250-68.

12 Martin R, Hocini M, Haïsaguerre M, Jaïs P, Sacher F. Ventricular Tachycardia Isthmus Characteristics: Insights from High-density Mapping. Arrhythm Electrophysiol Rev. 2019 Mar;8(1):54-9. 